\author{
Е. Л. Казаков ${ }^{1}$, А. Е. Казаков ${ }^{2}$, В. М. Решетник ${ }^{3}$ \\ ${ }^{1}$ Летная академия Национального авиационного университета, Кропивницкий, Украина \\ ${ }^{2}$ Харьковская государственная академия культуры, Харьков, Украина \\ ${ }^{3}$ Харьковский Национальный университет радиоэлектроники, Харьков, Украина
}

\title{
ВОЗМОЖНОСТИ ПОЛУЧЕНИЯ ПОЛЯРИЗАЦИОННЫХ МАТРИЦ РАССЕЯНИЯ ЦЕЛЕЙ ПРИ УПРАВЛЕНИИ ПОЛЯРИЗАЦИЕЙ ПОЛЯ АНТЕН РЛС
}

\begin{abstract}
Предметом изучения в статье являются процессы управления поляризацией поля антенных систем для решения прикладных задач распознавания радиолокационных целей. Целью является получение матричных выражений для пересчета элементов поляризационной матрицы рассеяния (ПМР) цели из произвольного базиса в ортогональный. Задачи: повышение скрытности и помехозащищенности работы РЛС; проанализировать существующий математический аппарат для получения матричных выражений; выбрать эффективный алгоритм его применения. Используемыми методами являются: математические модели оптимизации, методы решения матричных задач. Получены следующие результаты. В общем виде получены матричные выражения, позволяющие производить пересчет элементов ПМР цели, измеренных в произвольном поляризационном базисе РЛС, с учетом характеристик этого базиca, в линейный ортогональный базис. Выводы. Научная новизна полученных результатов состоит в следующем: получены матричные выражения позволяющие по измеренным при управлении поляризацией поля антенн РЛС, элементам ПМР цели и характеристикам данного поляризационного базиса, рассчитать элементы ПМР цели в ортогональном поляризационном базисе РЛС; при проведении расчетов необходимо учитывать возможные ошибки получения элементов ПМР в заданном ортогональном поляризационном базисе. Эти ошибки обусловлены приближением ортов разных поляризаций, на передачу и прием, друг к другу при управлении поляризацией антенн.
\end{abstract}

Ключевые слов а: радиолокационная цель, поляризационная матрица рассеяния цели, поляризационный измерительный базис, характеристики измерительного базиса.

\section{Введение}

Постановка проблемы. В настоящее время большое внимание уделяется распознаванию различных классов радиолокационных целей (РЛЦ) при использовании различных видов сигналов, в том числе и поляризационная матрица рассеяния (ПМР). Одновременно для повышения скрытности работы РЛС или с целью увеличения ее потенциала необходимо излучать зондирующие сигналы на произвольных эллиптических поляризациях. Помехозащищенность РЛС также требует в общем случае использования произвольного эллиптического базиса приемной антенны. В тоже время использование при распознавании РЛЦ элементов ПМР, измеренных при произвольном поляризационном базисе (ПБ) антенн, становится невозможным, так как обучение алгоритмов распознавания будет всегда проводиться в заранее известном и ортогональном базисе. Поэтому, получение ПМР в ортогональном ПБ по измеренным комплексным амплитудам отраженных сигналов при произвольных базисах передающей и приемной антенн РЛС становится актуальным. В статье в общем виде получены матричные выражения, позволяющие производить пересчет элементов ПМР цели, измеренных в произвольном ПБ РЛС, с учетом характеристик этого базиса, в линейный ортогональный базис. Рассмотрены три частных случая пересчета элементов ПМР цели при управлении поляризацией поля антенн РЛС.

Анализ последних исследований и публикаций. Возможностям и методам измерения элементов ПМР целей посвящено большое количество научной литературы. В частности эти вопросы подробно рассмотрены в [1 - 5]. Однако, в большинстве источни- ков приводятся примеры измерений элементов ПМР в ортогональных поляризационных базисах. При необходимости рассматриваются методы пересчета измеренных элементов ПМР цели из одного ортогонального измерительного базиса РЛС в другой $[1,2]$. В общем виде расчетные выражения для пересчета элементов ПМР цели, измеренных в ПБ при управлении поляризацией антенн РЛС в ортогональный базис, не получены.

Целью статьи является получение матричных выражений, позволяющих пересчитать измеренные при произвольных базисах передающей и приемной антенн РЛС элементы ПМР цели в ортогональный поляризационный базис.

\section{Основной материал}

Пусть необходимо получить ПМР цели в ортогональном линейном базисе (y, x). Здесь и в дальнейшем первый символ обозначает базис приемной антенны РЛС, а второй - передающей. Тогда матрица комплексных амплитуд отраженных сигналов $E_{0}^{(y)}$ через матрицу комплексных амплитуд излученных сигналов $E_{p}^{(x)}$ и ПМР объекта $S^{(y, x)}$ в базиce $(y, x)$ может быть записана в виде:

$$
E_{0}^{(y)}=S^{(y, x)} E_{p}^{(x)} .
$$

Допустим далее, что базис передающей антенны изменился, и излучение сигналов произошло в базисе $p\left(\vec{p}_{1}, \vec{p}_{2}\right)$, а прием отраженных сигналов осуществился в базисе $q\left(\vec{q}_{1}, \vec{q}_{2}\right)$ (рис. 1 ). В общем случае $\vec{p}_{1}, \vec{p}_{2}$ и $\vec{q}_{1}, \vec{q}_{2}$ могут быть произвольными комплексными числами. 
Передача
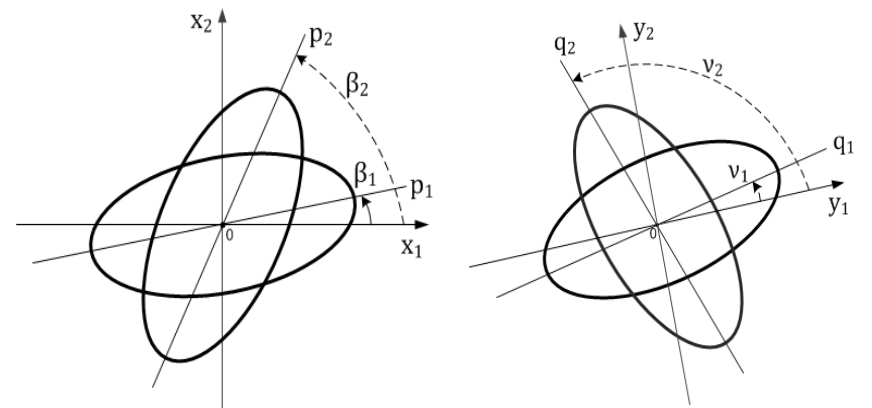

Рис. 1. Базис передающей и приемной антенн

Тогда матрицы комплексных амплитуд излученных и принятых сигналов с учетом изменения поляризационных базисов можно записать в виде:

$$
\begin{aligned}
& E_{p}^{(x)}=F_{p}^{(x, p)} E_{p}^{(p)}, \\
& E_{o}^{(y)}=F_{o}^{(y, q)} E_{o}^{(q)},
\end{aligned}
$$

где $F_{p}^{(x, p)}, F_{o}^{(y, q)}$ - матрицы перехода от базиса

$(\vec{p})$ к базису $(\vec{x})$ и от базиса $(\vec{q})$ к базису $(\vec{y})$.

Производя матричные преобразования (2) и используя их в (1), получим:

$$
E_{0}^{(q)}=F_{0}^{(y, q)^{-1}} S^{(y, x)} F_{p}^{([, p)} E_{p}^{(p)},
$$

или

$$
E_{0}^{(q)}=S^{(q, p)} E_{p}^{(p)}
$$

$$
\text { где } \quad S^{(q, p)}=F_{0}^{(y, q)^{-1}} S^{(y, x)} F_{p}^{(x, p)} .
$$

Тогда искомую матрицу в ортогональном базиce $(\vec{y}, \vec{x})$ можно из выражения (5) записать в виде:

$$
S^{(y, x)}=F_{o}^{(y, q)} S^{(q, p)} F_{p}^{(x, p)-1} .
$$

Таким образом, используя выражение (6) можно получить ПМР объекта в ортогональном базисе и в дальнейшем использовать ее для распознавания.

Для определения матриц пересчета $E_{o}^{(y, q)}$ и $E_{p}^{(x, p)}$ воспользуемся рис. 1. Из него следует, что базисные эллиптические поляризации на передачу характеризуются углами наклонения эллипсов поляризации $\beta_{1}, \beta_{2}$ и углами эллиптичности $\varepsilon_{1}, \varepsilon_{2}$, а на прием - углами наклона эллипсов поляризации $v_{1}$, $v_{2}$ и углами эллиптичности $\alpha_{1}, \alpha_{2}$ [5]. Тогда матрицы пересчета имеют вид:

$$
F_{0}^{(y, q)}=\left(\begin{array}{ll}
\cos v_{1} \cos \alpha_{1}- & \cos v_{2} \cos \alpha_{2}- \\
-j \sin v_{1} \sin \alpha_{1} & -j \sin v_{2} \sin \alpha_{2} \\
\sin v_{1} \cos \alpha_{1}+ & \sin v_{2} \cos \alpha_{2}+ \\
+j \cos v_{1} \sin \alpha_{1} & +j \cos v_{2} \sin \alpha_{2}
\end{array}\right),
$$

$$
F_{p}^{(p, x)}=\left(\begin{array}{cc}
\cos \beta_{1} \cos \varepsilon_{1}- & \cos \beta_{2} \cos \varepsilon_{2}- \\
-j \sin \beta_{1} \sin \varepsilon_{1} & -j \sin \beta_{2} \sin \varepsilon_{2} \\
\sin \beta_{1} \cos \varepsilon_{1}+ & \sin \beta_{2} \cos \varepsilon_{2}+ \\
+j \cos \beta_{1} \sin \varepsilon_{1} & +j \cos \beta_{2} \sin \varepsilon_{2}
\end{array}\right) .
$$

Обратная матрица $\left(F_{p}^{(p, x)}\right)^{-1}$ в соответствии с [4] имеет вид:

$$
=\frac{1}{D_{p}}\left(\begin{array}{cc}
\left(F_{p}^{(p, x)}\right)^{-1}= \\
=j \cos \beta_{2} \sin \varepsilon_{2} & +j \sin \beta_{2} \sin \varepsilon_{2} \\
-\sin \beta_{1} \cos \varepsilon_{1}- & \cos \beta_{1} \cos \varepsilon_{1}- \\
-j \cos \beta_{1} \sin \varepsilon_{1} & -j \sin \beta_{1} \sin \varepsilon_{1}
\end{array}\right),
$$

Рассмотрим в качестве примеров некоторые возможные частные случаи управления поляризацией поля антенн РЛС и проанализируем получаемые при этом выражения для матриц пересчета $F_{o}^{(y, q)}$ и $F_{p}^{(x, p)}$.

1. Если базис приемной антенны q является линейным ортогональным, но его базовые поляризации не совпадают с ортами $\vec{y}_{1}$ и $\vec{y}_{2}$, то есть $v_{1} \neq 0$, а $v_{2}-v_{1}=90^{\circ}$ (рис. 2), то матрица преобразования $F_{0}^{(y, q)}$ превратится в обычную матрицу поворота

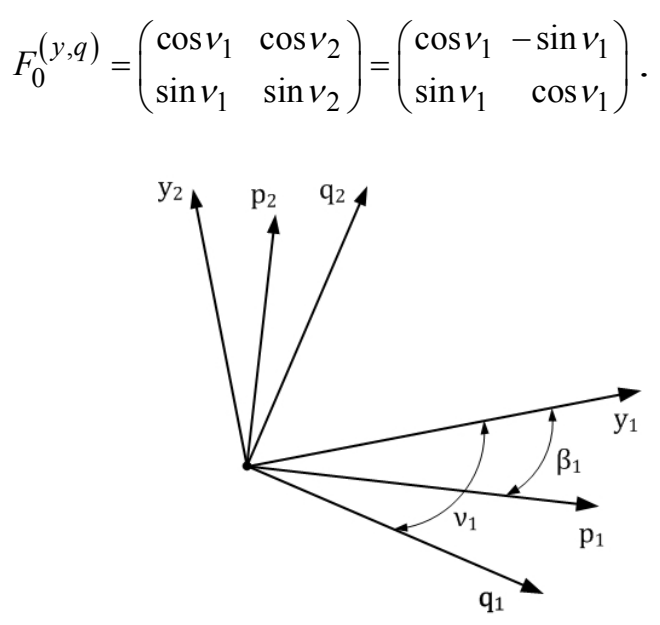

Рис. 2. Базис приемной антенны $q$

Легко заметить, что, если базис передающей антенны $p$ будет ортогональным $\left(\beta_{1} \neq 0^{\circ}, \beta_{2}-\beta_{1}=90^{\circ}\right)$, то матрица преобразования $\left(F_{p}^{(p, x)}\right)^{-1}$ будет также представлять собой матрицу поворота 


$$
\left(F_{p}^{(p, x)}\right)^{-1}=\left(\begin{array}{cc}
\sin \beta_{2} & -\cos \beta_{2} \\
-\sin \beta_{1} & \cos \beta_{1}
\end{array}\right)=\left(\begin{array}{lc}
\cos \beta_{1} & \sin \beta_{1} \\
-\sin \beta_{1} & \cos \beta_{1}
\end{array}\right) .
$$

Тогда выражение для получения ПМР в базиce $(y, x)$ примет вид:

$$
S^{(y, x)}=\left(\begin{array}{cc}
\cos v_{1} & -\sin v_{1} \\
\sin v_{1} & \cos v_{1}
\end{array}\right) S^{(q, p)}\left(\begin{array}{cc}
\cos \beta_{1} & \sin \beta_{1} \\
-\sin \beta_{1} & \cos \beta_{1}
\end{array}\right) .
$$

Полученное соотношение особенно наглядно, когда базис передающей антенны (p) представляет собой повернутый на некоторый угол базис (х) и базис приемной антенны (q) также повернутый на такой же угол базис (у). Тогда соотношение (12) будет представлять собой обычное преобразование ПМР объекта при повороте исходного базиса (y, х). Для случая, когда базис приемной антенны (у) будет совмещен с базисом передающей антенны (х), выражение (12) превращается в хорошо известное соотношение [1].

2. Рассмотрим случай, когда базис передающей антенны $p$ и базис приемной антенны $q$ являются круговыми. Тогда, чтобы определить матрицу рассеяния $\mathrm{S}^{(\mathrm{y}, \mathrm{x})}$ в искомом линейном базисе $(\mathrm{y}, \mathrm{x})$ необходимо вычислить матрицы пересчета $F_{0}^{(y, q)}$ и $\left(F_{p}^{(p, x)}\right)^{-1}$. Для определенности при нахождении матрицы $F_{0}^{(y, q)}$ положим, что $v_{1}=0^{\circ}, v_{2}=90^{\circ}$, $\alpha_{1}=-45^{\circ}, \alpha_{2}=45^{\circ}$. Тогда

$$
F_{0}^{(y, q)}=\frac{1}{\sqrt{2}}\left(\begin{array}{cc}
1 & -j \\
-j & 1
\end{array}\right) .
$$

При отыскании $\left(F_{p}^{(p, x)}\right)^{-1}$ положим $\beta_{1}=0^{\circ}$, $\beta_{2}=90^{\circ}, \varepsilon_{1}=-45^{\circ}, \varepsilon_{2}=45^{\circ}$. Тогда имеем

$$
\left(F_{p}^{(p, x)}\right)^{-1}=\frac{1}{\sqrt{2}}\left(\begin{array}{cc}
1 & j \\
j & 1
\end{array}\right) \text {. }
$$

В этом случае выражение для определения искомой матрицы $\mathrm{S}^{(\mathrm{y}, \mathrm{x})}$ примет вид:

$$
S^{(y, x)}=\frac{1}{2}\left(\begin{array}{cc}
1 & -j \\
-j & 1
\end{array}\right) \times S^{(q, p)}\left(\begin{array}{ll}
1 & j \\
j & 1
\end{array}\right) .
$$

Полученное соотношение (15) представляет собой хорошо известное преобразование ПМР объекта из кругового базиса в линейный.

Таким образом, из рассмотренных примеров видно, что полученные результаты не противоречат результатам, описанным в работе [1]. Однако когда в РЛС используются произвольные ПБ антенн, следует обращать внимание на ошибки при определении элементов ПМР объекта в искомом базисе (y, х), возникающие при отклонении базисных векторов от ортогональных.

3. Рассмотрим в качестве примера случай, когда ПБ передающей антенны (р) является линейным, но не ортогональным. Положение базисных векторов определяется углами $\beta_{1}$ и $\beta_{2}$, а прием отраженного сигнала производится в линейном ортогональном базисе, совпадающем с базисом (х) (рис. 3). Необходимо определить ПМР объекта в базисе (х) при условии, что она измеряется в базисе $(\mathrm{x}, \mathrm{p})$.

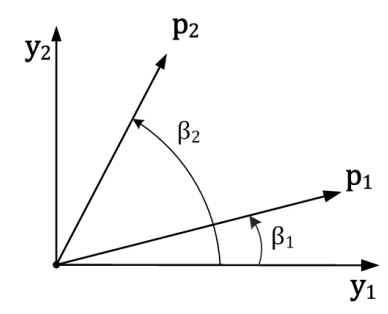

a - Передача

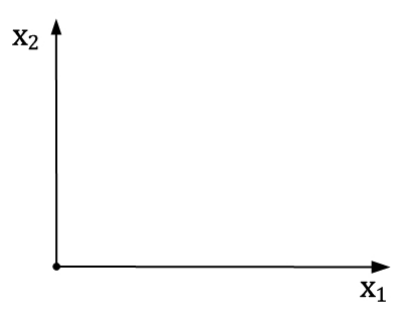

б - Прием
Рис. 3. Линейный поляризационный базис передающей антенны $(p)$

Для этого найдем аналитические выражения для матриц пересчета $F_{0}^{(y, q)}$ и $\left(F_{p}^{(p, x)}\right)^{-1}$, а также воспользуемся выражением (9). Очевидно, что матрица пересчета $F_{0}^{(y, q)}$ равна единичной матрице: $F_{0}^{(y, q)}=$ Е. Для нахождения матрицы пересчета $\left(F_{p}^{(p, x)}\right)^{-1}$ воспользуемся рис. 3 , а. Тогда

$$
\begin{gathered}
\left(F_{p}^{(p, x)}\right)=\left(\begin{array}{ll}
\cos \beta_{1} & \cos \beta_{2} \\
\sin \beta_{1} & \sin \beta_{2}
\end{array}\right), \\
\left(F_{p}^{(p, x)}\right)^{-1}=\frac{1}{\sin \left(\beta_{2}-\beta_{1}\right)}\left(\begin{array}{cr}
\sin \beta_{2} & -\cos \beta_{2} \\
-\sin \beta_{1} & \cos \beta_{1}
\end{array}\right) .
\end{gathered}
$$

Таким образом, получим окончательное выражение для нахождения матрицы

$$
\begin{aligned}
& S^{(x)}=S^{(x, p)}\left(F_{p}^{(p, x)}\right)^{-1}=\frac{1}{\sin \left(\beta_{2}-\beta_{1}\right)} \times \\
& \times\left(\begin{array}{ll}
\dot{S}_{11}^{(x, p)} & \dot{S}_{12}^{(x, p)} \\
\dot{S}_{21}^{(x, p)} & \dot{S}_{22}^{(x, p)}
\end{array}\right) \times \times\left(\begin{array}{cc}
\sin \beta_{2} & -\cos \beta_{2} \\
-\sin \beta_{1} & \cos \beta_{1}
\end{array}\right) .
\end{aligned}
$$

Из анализа соотношения (17) видно, что в рассмотренном примере при $\beta_{2}$ стремящемся к $\beta_{1}$ матрица пересчета $\left(F_{p}^{(p, x)}\right)^{-1}$ становится плохо обусловленной, что может привести к большим ошибкам определения элементов ПМР S(x) в искомом базисе $(\mathrm{x})$ даже при незначительных отклонениях углов $\beta_{1}$ и $\beta_{2}$ от истинных. Величины этих ошибок также необходимо учитывать.

\section{Выводы}

Полученные матричные выражения позволяют по измеренным при управлении поляризацией поля антенн РЛС, элементам ПМР цели и характеристикам данного ПБ, рассчитать элементы ПМР цели в 
ортогональном ПБ РЛС. Однако, при проведении расчетов необходимо учитывать возможные ошибки получения элементов ПМР в заданном ортогональ- ном ПБ. Эти ошибки обусловлены приближением ортов разных поляризаций, на передачу и прием, друг к другу при управлении поляризацией антенн.

\section{СПИСОК ЛІТЕРАТУРИ}

1. Канарейкин Д. Б. Поляризация радиолокационных сигналов / Д. Б. Канарейкин, Н. Ф. Павлов, В. А. Потехин. - М.: Сов. радио, 1966. -440 с.

2. Распознавание радиолокационных целей по сигнальной информации. Монография / Е. Л. Казаков, Д. Г. Васильев, А. Е. Казаков, Д. Н. Рыжов, А. В. Коломийцев; под ред. Е. Л. Казакова. - Х.: КП «Міська друкарня». 2010. - 232 с.

3. Казаков Е. Л. Поляризационные методы распознавания радиолокационных целей / Е. Л. Казаков, А. Е. Казаков // Прикладная радиоэлектроника. - Харьков : ХНУРЕ, 2002 - Т. 1,. № 2. - С. 155 - 163.

4. Радиоэлектронные системы. Основы построения и теория. Справочник / Под ред. Я. Д. Ширмана. - М.: Радиотехника, 2007. $-510 \mathrm{c}$.

5. Казаков Е. Л. Распознавание целей по сигнальной информации в однопозиционных и многопозиционных локаторах / Е. Л. Казаков, А. Е. Казаков, А. В. Коломийцев; под ред. Е. Л. Казакова. - Х.: ХУВС, 2015. - 459 с.

\section{REFERENCES}

1. Kanareikin, D.B., Pavlov, N.F. and Potekhin, V.A. (1966), Polarization of radar signals, Sov. radio, Moscow, 440 p.

2. Kazakov, E.L., Vasiliev, D.G., Kazakov, A.E., Ryzhov, D.N. and Kolomiytsev, A.V. (2010), Recognition of radar targets based on signal information, edited by Kazakov, E.L., Miska drukarnya, Kharkiv, 232 p.

3. Kazakov, E.L. and Kazakov, A.E. (2002), "Polarization methods for recognizing radar targets", Applied radioelectronics, KHNURE, Kharkiv, Vol. 1,. No. 2, pp. 155-163.

4. Shirman, Ya.D. (2007), Radioelectronic systems. Fundamentals of construction and theory, Moscow, Radio engineering, $510 \mathrm{p}$.

5. Kazakov, E.L., Kazakov, A.E. and Kolomiytsev A.V. (2015), Recognition of the targets for signal information in singleposition and multi-position locators, KhUPS, Kharkiv, 459 p.

Рецензент: д-р техн. наук, проф. В. М. Биков, Харківський національний університет імені В. Н. Каразіна, Харків Received (Надійшла) 14.03.2018 Accepted for publication (Прийнята до друку) 28.04.2018

\section{Можливості отримання поляризаційних матриць розсіяння цілей при управлінні поляризацією поля антен РЛС}

\section{Є. Л. Казаков, О. Е. Казаков, В.М. Решітник}

Предметом вивчення в статті $€$ процеси управління поляризацією поля антенних систем для вирішення прикладних завдань розпізнавання радіолокаційних цілей. Метою $є$ отримання матричних виразів для перерахунку елементів поляризаційної матриці розсіювання (ПМР) цілі з довільного базису в ортогональний. Завдання: підвищення скритності і перешкодозахищеності роботи РЛС; проаналізувати існуючий математичний апарат для отримання матричних виразів; вибрати ефективний алгоритм його застосування. Використаними методами $\epsilon$ : математичні моделі оптимізації, методи вирішення матричних задач. Отримані наступні результати. У загальному вигляді отримані матричні вирази, що дозволяють здійснити перерахунок елементів ПМР цілі, виміряних в довільному поляризаційному базисі РЛС, 3 урахуванням характеристик цього базису, в лінійний ортогональний базис. Висновки. Наукова новизна отриманих результатів полягає в наступному: отримані матричні вирази, що дозволяють по виміряним при управлінні поляризацією поля антен РЛС, елементам ПМР цілі і характеристикам даного поляризаційного базису, розрахувати елементи ПМР цілі в ортогональному поляризаційному базисі РЛС; при проведенні розрахунків необхідно враховувати можливі помилки отримання елементів ПМР в заданому ортогональному поляризаційному базисі. Ці помилки обумовлені наближенням ортів різних поляризацій, на передачу і прийом, один до одного при управлінні поляризацією антен.

Ключов і слов а: радіолокаційна ціль, поляризаційна матриця розсіювання цілі, поляризаційний вимірювальний базис, характеристики вимірювального базису.

\section{Possibilities of obtaining polarization scattering matrixs for polarization of radar antenna field \\ Ye. Kazakov, O. Kazakov, V. Reshetnik}

The subject of the study in the article are the processes of controlling the polarization of the field of antenna systems for solving applied problems of radar target recognition. The goal is to obtain matrix expressions for recalculating the elements of the polarization scattering matrix (PMR) of a target from an arbitrary basis to an orthogonal one. The tasks: increase stealth and noise immunity of the radar; analyze the existing mathematical apparatus for obtaining matrix expressions; choose an effective algorithm for its application. The methods used are: mathematical optimization models, methods for solving matrix problems. The following results are obtained. Generally, matrix expressions are obtained that allow the recalculation of the target PMR elements measured in an arbitrary polarization basis of the radar, taking into account the characteristics of this basis, into a linear orthogonal basis. Conclusions. The scientific novelty of the obtained results consists in the following: matrix expressions are obtained that allow us to calculate the target PMR elements in the orthogonal polarization basis of the radar from the radar antenna fields, target PMR and characteristics of the polarization basis measured during the polarization control; when making calculations it is necessary to take into account possible errors in obtaining PMR elements in a given orthogonal polarization basis. These errors are due to the approximation of the units of different polarizations, to transmission and reception, to each other when controlling the polarization of the antennas.

Keywords : radar target, polarization target scattering matrix, polarization measuring basis, characteristics of the measuring base. 